\title{
Was wir weglassen sollten
}

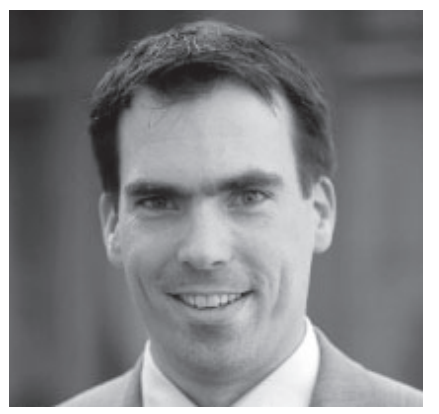

VON SVEN LÜNGEN

Sven Lüngen ist Sozialpädagoge und Betriebswirt. Er ist Geschäftsführer des Alexander-Stifts, einem großen Altenhilfeträger in Baden-Württemberg, Tochtergesellschaft der Diakonie Stetten.

www.alexander-stift.de

\author{
Nicht das "Mehr", sondern ein verantwortungsvolles \\ "Weniger" gilt es zu diskutierten, meint der Autor in \\ seinem Plädoyer für eine nachhaltige Wirksamkeit \\ Sozialer Arbeit und er stellt mit Peter Drucker die Frage: \\ "Was von all dem, was wir heute tun, würden wir nicht \\ mehr beginnen, wenn wir es nicht schon täten?»
}

In allen Bereichen der Sozialen Arbeit sind die Rufe laut und klar zu hören: »Mehr Geld! « und »Mehr Personal! « So lautet das Credo von vielen engagierten Sozialarbeitern und Sozialmanagern, aber auch von Sozialpolitikern und Lobbyisten. Sie setzen sich für bestehende Leistungen und Institutionen ein und wollen jene im Interesse unterstützungsbedürftiger, hilfesuchender und pflegebedürftiger Menschen auch in Zukunft absichern.

Exemplarisch sei für diese Feststellung auf die Forderung eines großen Altenhilfeträgers in Süddeutschland verwiesen. Im Hinblick auf die von Gesundheitsminister Bahr angekündigte Pflegereform steht der Geschäftsführer dieser Unternehmensgruppe für deutlich bessere Rahmenbedingungen in der Pflege ein. Er fordert »mehr Personal für Pflege und Betreuung, bessere Qualifikationsmöglichkeiten und eine nachhaltige Finanzierung der Pflegeleistungen «.

Die Absicherung solidarisch finanzierter Leistungen ist ein richtiges und wichtiges Anliegen. Vor dem Hintergrund demografischer und gesellschaftlicher Entwicklungen ist jedoch infrage zu stellen, ob dies mit einer Forderung nach »Mehr« wirklich nachhaltig gelingen kann. (Zudem lässt der wachsende Fachkräftemangel die Diskussion um mehr Personal zu einer unrealistischen Forderung werden. - Dies wäre ein Thema für einen weiteren Beitrag.)

Bei der Finanzierung Sozialer Arbeit setzen die Kostenträger seit Jahren immer wieder auf noch vorhandene Effizienzpotenziale bei den Leistungserbringern. Reale Sach- und Lohnkostensteigerungen spiegeln sich nicht in Entgeltsteigerungen wieder. Prospektive Kostenentwicklungen oder unternehmerische Risiken werden nicht umfassend berücksichtigt. Zwar stellen Urteile des Bundessozialgerichts - in der Altenhilfe zum Beispiel B3P 9/07 R vom 29. Januar 2009 - auf Eigenkapitalverzinsung und unternehmerisches Risiko ab, auf die Praxis von Entgeltverhandlungen aber haben diese Urteile bisher nur wenig Einfluss.

Folglich sind das Verhältnis von Qualität, Leistungsansprüchen und steigenden Anforderungen einerseits zu der Form und dem Maß der Refinanzierung andererseits immer mehr ins Ungleichgewicht geraten. Trotz jahrelanger Bemühungen ist es den Leistungsträgern bisher nicht gelungen, ein reales "Mehr « zu erstreiten. Die in Entgeltverhandlungen realisierten Steigerungen sind unter dem Strich von Inflation und wachsenden Anforderungen immer ein "Weniger« gewesen.

Konsequenterweise bedeutet dies, dass Soziale Arbeit in fast allen Bereichen immer noch "effizienter " gemacht werden muss, wenn inhaltliche und formale Anforderungen nicht überdacht werden. Es muss schneller gearbeitet werden und es gilt, mit immer weniger Arbeitszeit weiter steigenden qualitativen Ansprüchen und zusätzlichen Aufgaben gerecht zu werden. Dieser Effizi- 
enzdruck ist gerade für kleine Einrichtungen im Besonderen zu spüren.

\section{Wir sitzen in der Effizienzfalle}

Damit sind wir an einem Punkt angekommen, an dem klar wird, dass wir die Effizienzschraube bereits zu weit gedreht haben. Die psychischen und physischen Belastungen der Mitarbeitenden sind enorm hoch. Immer weniger Kolleginnen und Kollegen schaffen es, ihre Aufgaben zeitgerecht abzuarbeiten und dabei auch noch glücklich und gesund zu bleiben. Das Gegenteil ist der Fall: Krankheits- und Fehlerquoten schnellen hoch!

Wir sitzen in der Effizienzfalle und müssen erkennen, dass jegliches weitere Anziehen der Effizienzschraube bei alle geforderten Aufgaben abzuarbeiten. Mitarbeitende sind überfordert, wenn es nicht eine adäquate und ausreichende Form der Unterstützung für sie gibt. In immer mehr Situationen entscheidet deshalb der einzelne Mitarbeiter, welche Aufgaben im Einzelfall nicht erfüllt werden. Rationierung ist somit nicht mehr ausschließlich eine Sorge bezüglich der zukünftigen Versorgung in Sozialeinrichtungen, sondern sie ist bereits heute eine Realität, eine in vielen Fällen ungesteuerte und auf der Grundlage von Werten und Normen des jeweiligen Mitarbeiters entschiedene Form der Rationierung.

Dieses »Nichterfüllen« von Leistungen und die Tatsache, dass es an vielen Stellen für die Menschen unbemerkt bleibt (und nicht von besonderer Be-
" Die vorhandenen Ressourcen reichen schlicht nicht aus, um alle geforderten Aufgaben zu bewältigen " getriebene Effektivitätsdiskussion ergänzend hinzustellen. Wir müssen darüber sprechen, was zielführend ist und welche Leistungen wirklich einen Beitrag zur Erfüllung unserer Aufgaben leisten. Wir müssen eindeutige Prioritäten setzen und den Mut haben, sie angemessen zu vertreten. Wenn wir nicht alles erfüllen können, was an Erwartungen und Anforderungen an uns gestellt wird, dann müssen wir uns primär an den Wünschen und Vorstellungen der Menschen orientieren, die sich uns anvertrauen.

Verantwortungsvoll mit den Ressourcen und den uns für unsere Arbeit überlassenen Mitteln umzugehen bedeutet für mich, sie zielgerichtet für die Menschen einzusetzen. Damit muss der Fokus von den Struktur- und Prozessqualitäten weg auf die Ergebnisqualitäten gelenkt werden. "Wohlbefinden", "Zufriedenheit" und weitere Aspekte der Lebensqualität werden somit zu wichtigen Navigationshilfen für einen angemessenen Mitteleinsatz.

Und schlussendlich müssen wir alles, was unter diesem Ergebnisaspekt keinen direkten oder mittelbaren Beitrag leistet, infrage stellen. Wir dürfen in der Sozialen Arbeit nicht länger überlegen, was alles für die Menschen wünschenswert ist. Wir müssen mit ihnen sprechen und den Mut haben, ihnen zuzuhören. Dies bietet die Chance, Rationierungen im Sinne der Menschen auszugestalten und uns auf die wesentlichen und wichtigen Aspekte zu konzentrieren.

Auf diesem Wege wird es möglich, einzelne Aufgaben und Tätigkeiten zu reduzieren oder ganz wegzulassen. Peter Drucker hat uns schon in den 1960er Jahren eine hierfür sinnvolle Frage zu Reflexion mitgegeben: "Was von all dem, was wir heute tun, würden wir nicht mehr beginnen, wenn wir es nicht schon täten?«. Fredmund Malik hat aus diesem Gedanken die »Systematische Müllabfuhr« entwickelt und als ein wichtiges Managementwerkzeug herausgestellt (Führen, Leisten, Leben. S. 359 ff.). Mit diesem Hilfsmittel können wir Ressourcen neu verteilen. Es gilt, unseren Ressourceneinsatz in der direkten Tätigkeit für die Menschen zu hinterfragen, aber auch die administrativen Bereiche zu untersuchen, welche Beiträge sie für die Menschen leisten.

Ich schlage vor: Lassen Sie uns alles das weglassen, was sinnvoll vertretbar 
ist und keinen ausreichenden Beitrag für die Menschen bringt!

Ein Beispiel hierfür ist die zwar notwendige, aber ausufernde Dokumentation sozialer Dienstleitungen. Wenn der Eindruck entsteht, dass die Dokumentation wichtiger als die erbrachte Leistung ist, dann sind wir an einem riskanten Punkt Sozialer Arbeit angekommen. Es ist keine Frage: Wir müssen das dokumentieren, was wir für eine fachlich fundierte Arbeit brauchen - jedoch keine Zeile mehr als notwendig!

Auch eine Mentalität der übermäßigen Risikovermeidung bietet Potenzial zum »Weglassen « und Ressourcensparen. Dürfen wir hilfe- und unterstützungsbedürftigen Menschen noch $\mathrm{Ri}$ siken des Alltages zumuten oder müssen wir jegliche Risiken des täglichen Lebens auf das denkbar geringste Maß zurückführen? Hygienevorschriften, die sich in der Altenhilfe am Krankenhausstandard orientieren anstatt an einer durchschnittlichen häuslichen Situation, bieten Ansätze für Diskussionen. Wenn ein alter Mensch mit über 90 Jahren in seiner eigenen Wohnung lebt, gelten für ihn wie für jeden anderen Bürger keine besonderen Schutzvorschriften. Mit dem Tag seines Umzugs in ein Pflegeheim wird er auf dem Niveau von Krankenhausstandards versorgt.

In den administrativen Bereichen und im Sozialsystem als Ganzes gibt es ebenfalls Einsparpotenziale. Es wäre spannend einmal zu diskutieren, ob die Verhandlung von Pflegesätzen wirklich einen Beitrag für die Menschen leistet. Sind die notwendigen Ressourcen zur Verhandlung wirklich angemessen eingesetzt? Was resultiert, wenn das Verhandlungsparadigma durch einfachere, pragmatischere Mechanismen ersetzt würde?

Wären bei den hier exemplarisch genannten Aspekten tatsächlich Einsparungen erreicht, würde auf diesem Wege Wünschenswertes auch zu Machbarem. Nicht das, was alles inhaltlich wünschenswert ist, sondern das, was aus Sicht der Menschen wichtig und für die Leistungsträger refinanzierbar ist, würde zukünftig in den Fokus genommen.

\section{Ausblick: Weniger ist gerechter}

Ein Blick in die kommunalen Haushalte macht deutlich, warum ein "Mehr für die Menschen«, das von Leistungs- erbringern, Politik und Verwaltungen gewünscht wird, vor dem Hintergrund demografischer Entwicklungen kaum finanzierbar ist.

In den nächsten Jahren ist in den meisten Regionen Deutschland ein $\mathrm{Zu}-$ wachs von Sozialleistungsempfängern zu erwarten. Wenn also die Sozialbudgets bereits hierdurch enorm belastet werden, ist doch die Frage, wie dann auch noch Leistungs- und Entgeltsteigerungen wirtschaftlich dargestellt werden können.

Auch die aktuell gute wirtschaftliche Entwicklung in Deutschland, die damit verbundene Entlastung der öffentlichen Haushalte und die zusätzlichen Mittel für die Sozialversicherungssysteme werden diese Effekte nicht im Ansatz kompensieren können. Insofern scheint mir nicht die Frage entscheidend zu sein, ob sich der Trend eines Weniger weiter fortsetzen wird, sondern ausschließlich die Frage, an welchen Stellen es zu Kürzungen kommt. Ich bin davon überzeugt, dass ohne fachlich fundierte Positionen aus Sicht der Sozialen Arbeit und im In- teresse der Menschen rein monetär getriebene Entscheidungen aus Politik und Verwaltung uns mit noch schwierigeren Realitäten konfrontieren werden.

Insofern muss eine Effektivitätsdiskussion geführt werden. Sie kann dazu beitragen, dass an den richtigen Stellen gekürzt wird, es bei den Ergebnis-Qualitäten keine Abstriche gibt, sondern sie im günstigsten Fall sogar besser werden.

Der hier zur Diskussion gestellte Ansatz führt überdies zu mehr Generationengerechtigkeit. Sollte die Annahme richtig sein, dass die für jeden einzelnen Leistungsempfänger zur Verfügung stehenden Ressourcen durchschnittlich sinken, dann wäre ein Mehr für die heutige Generation ein zusätzliches Weniger für die Menschen, die künftig auf Hilfe und Unterstützung angewiesen sind.

Ein "Weniger" im Hier und Heute wird sich als ein zukünftiges "Mehr" herausstellen und einen Beitrag für die nachhaltige Absicherung Sozialer Arbeit leisten. Dies allerdings ist ein "Mehr", um das ich gerne diskutieren und ringen will.

\section{Systematische Müllabfuhr - ein Beispiel}

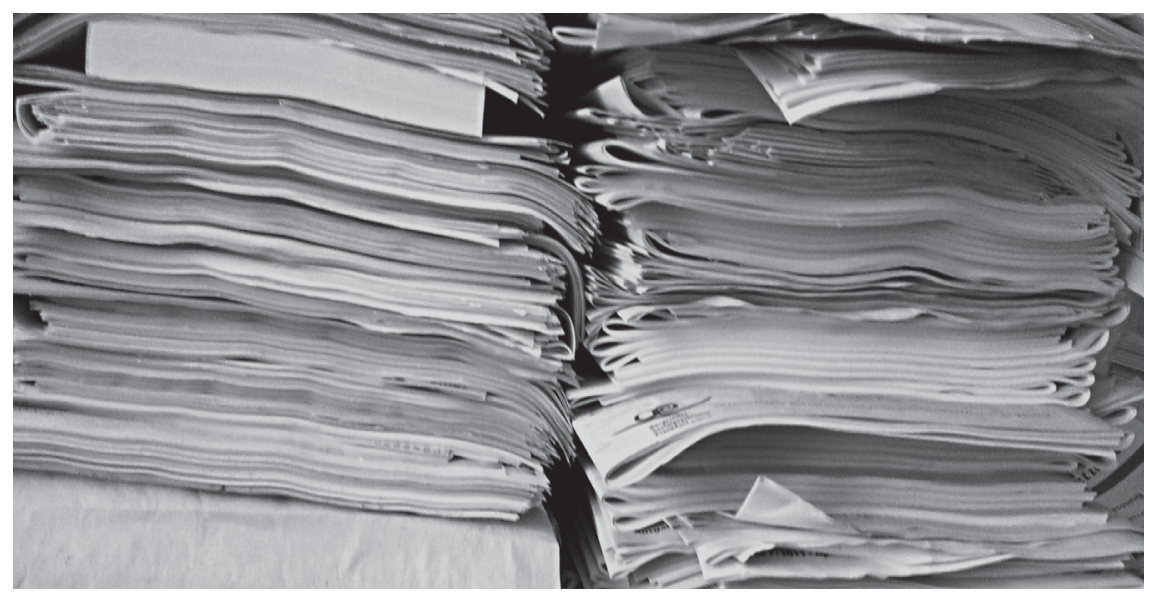

"Der spätere Lord Marks, einer der Gründer der noch immer sehr erfolgreichen englischen Warenhauskette, Marks \& Spencer, ging immer wieder in die Läden, um das Geschäft zu beobachten. Dabei fiel ihm auf, wie viel Papier von den Verkäuferinnen und Angestellten bewegt wurde. Daraufhin gab er die Weisung aus, alles Papier im Kundenverkehr zu eliminieren, außer die Quittung. Alle swussten natürlich ganz genau, dass das nicht ging, dass man ohne diese Papiere das Geschäft nicht betreiben könne usw. Marks blieb konsequent, und das Papier wurde eliminiert. Das einzige wirkliche Problem, das man nicht eliminieren konnte, war die Tatsache, dass man diese Prozedur alle drei Jahre wiederholen musste, weil das Papier schon wieder da war."

Quelle: Fredmund Malik: Systematische Müllabfuhr. Eine weithin unbekannte, aber sehr wirksame Idee. Malik Management Letter 7/95. 